\title{
Ciberbullying desde la perspectiva de la violencia hacia la identidad a través de las TIC
}

\author{
Marcelo Humberto Rioseco Pais ${ }^{1}$ \\ Universidad Católica del Maule \\ Katherine Morgado Gallardo ${ }^{2}$ \\ Universidad Católica del Maule
}

\begin{abstract}
Resumen: El siguiente artículo utiliza un cambio de mirada para definir el problema del bullying y del ciberbullying en la escuela, entendiendo la violencia escolar como un problema sistémico que involucra a los actores que participan directa o indirectamente en situaciones de maltrato, como sujetos interrelacionados mediante la construcción de sus identidades. Para tal efecto, se contextualiza, en primer lugar, el ciberbullying en el tipo de sociedad actual, determinada por el uso de TIC; en segundo lugar, se describen algunos problemas asociados a los conceptos de bullying y ciberbullying; luego se reflexiona en torno al concepto de violencia y su impacto en la conformación de la identidad; para, finalmente, describir la manera cómo se pone de manifiesto el ciberbullying y la violencia escolar a través del uso de las TIC.

Palabras clave: Ciberbullying, identidad, maltrato escolar, violencia, aprendizaje de la violencia.
\end{abstract}

Abstract: This article takes a different approach towards the definition of the issues of bullying and cyberbullying at school: we understand school violence as a systemic problem encompassing all direct or indirect players as individuals that are interrelated through the construction of their identities. For that purpose, we first contextualize cyberbullying in our current society, where the use of ICTs is pervasive. Then we describe some problems associated to the concepts of bullying and cyberbullying, followed by a reflection around the concept of violence and its impact on the process of forming one's identity. Finally, we describe ways in which the phenomena of cyberbullying and school abuse are brought to light through the use of ICTs.

Keywords: Cyberbullying, identity, school maltreatment, violence, learning of violence.

\section{Introducción}

Las implicancias de la transformación cultural provocada por la masificación de las tecnologías de la información y de la comunicación (TIC) en el último periodo histórico, ha conllevado a situarnos en nuevos y complejos escenarios, los cuales no involucran únicamente los marcos estructurales que sostienen a la sociedad, sean políticos, económicos, productivos o institucionales, si no que evidencian una nueva forma de interacción humana y de construcción social de la realidad, basada en las dinámicas virtuales que hoy es posible sostener gracias al acceso y al uso masivo de internet y de las redes sociales. Esta "virtualización" de las relaciones y por ende de las identidades personales atraviesa hoy en día, de forma transversal los diversos planos de la experiencia cotidiana, haciéndonos asimilar la magnitud del alcance, de lo que hoy muchos reconocen como una nueva revolución industrial.

\footnotetext{
${ }^{1}$ Master en Educación y TIC (UOC). Doctor en Educación de la Universidad de Alicante (España). Académico de la Facultad de Ciencias de la Educación de la Universidad Católica del Maule (Chile), Su línea de investigación es el uso de TIC en el la expresión del género a través del uso de las tecnologías.

${ }^{2}$ Psicóloga (ULS). Magister en Gestión Educacional (UCEN). Académica Departamento de Psicología, Facultad de Ciencias de la Salud de la Universidad Católica del Maule (Chile). Su línea de investigación es Convivencia y Violencia Escolar.
} 
En nuestro país, se ha evidenciado que un segmento importante de la población cuenta con acceso a internet, ya sea de forma directa o a través de recursos y medios facilitados por el entorno social, laboral o escolar. En el año 2014, según cifran de la Subsecretaria de Telecomunicaciones, el 66\% de la población declaraba contar con acceso permanente a la red (12 millones de personas aproximadamente). Por otro lado, un $62 \%$ de los hogares cuenta con acceso directo, un $27 \%$ más que el promedio de los países latinoamericanos, viéndose concentrado este acceso en las zonas urbanas por sobre las zonas rurales. En este sentido, en función de la distribución etaria, respecto al acceso y utilización de redes, esta se encuentra concentrada en personas jóvenes menores de 29 años (Rivera, J., Lima, J. \& Castillo, E., 2014).

Justamente, para estas generaciones, el escenario vivencial en el cual se han desarrollado, anclado la cultura de la inmediatez, del consumismo y de la hiperconectividad, ha implicado además una apropiación distintiva de los vínculos, en donde:

El proceso de producción se semiotiza y la formación del sistema nervioso digital implica y conecta la mente, el psiquismo social, los deseos y las esperanzas, los miedos y la imaginación. Por ello tenemos que ocuparnos de la producción semiótica, del cambio lingüístico y cognitivo. (Bifo, 2003).

De este modo, las diversas problemáticas sociales ya existentes, emergen hoy en día, también desde la plataforma y dinamismo que otorgan la utilización masiva de la red, evidenciando no solo el acceso existente, sino también el exceso beligerante de su utilización, casi como mecanismo de canalización del enajenamiento y de la fragmentación afectiva de los sujetos hacia el entorno y hacia los otros. Así, muchas de estas problemáticas, como la violencia y las variadas formas de discriminación social, por ejemplo, han adquirido nuevas formas, y dimensiones de expresión, sustento y reproducción.

El análisis de la violencia, entonces, comprendida desde el seno de la institución escolar, como institución que no solo recrea las formas de violencia estructural del sistema, sino que es además portadora y generadora de formas específicas de violencia, no es nuevo. El fenómeno de matonaje y acoso escolar, alusivo al concepto de bullying (Olweus, 1993), está representado y equiparado en esta era digital, en el fenómeno del ciberbullying, en donde las formas de maltrato, abuso, acoso y menoscabo se trasladan y se mantienen a través del uso de las tecnologías de la información y de la comunicación. En nuestro país, las agresiones reportadas a través de medios tecnológicos, según los datos entregados por la Tercera Encuesta Nacional de Violencia en el Ámbito Escolar (Ministerio del Interior, 2009), indicaron que un 10,6\% de los estudiantes declaró haber sido agredido por medio de internet y un 6,4\% admitió de igual modo, agredir a través de este medio. Tal como en el bullying, las consecuencias negativas para el niño, niña o adolescente que lo sufre, son complejas, sobre todo a nivel de desarrollo socio-emocional y de la salud mental, reportándose, inclusive, en los casos de victimización prolongada, ideación, intentos y concreción suicida (Rigby, 1996).

\section{Bullying y ciberbullying: conceptos problemáticos}

"Ciberbullying" es un término compuesto, utilizado, originalmente, en el idioma inglés e incorporado como anglicismo en el español. Ciber o cyber, indica 
relación con redes informáticas y bullying (de bull o "toro" y, luego, de bully o "matón") significa acoso. En términos generales, bullying se refiere a una forma de maltrato que se produce entre escolares, de manera reiterada y a lo largo del tiempo, donde, además, existe asimetría de fuerza o de poder. Generalmente, se utiliza el término bullying como sinónimo de violencia escolar (Del Barrio, Martín, Almeida, Barrios, 2003), aun cuando las dinámicas de bullying se fundamentan en cuatro características específicas: que sea entre pares, que sea frecuente y duradero, que exista un desequilibrio de poder y que esté presente la intencionalidad de hacer daño (Olweus, 1993)

En lo que se refiere al ciberbullying, para Joaquín Mora-Merchán "se ha convertido en un problema emergente dentro de nuestro sistema educativo" (2008), ya que en la actualidad los estudiantes utilizan frecuentemente las TIC para maltratar a sus compañeros. Por supuesto que la violencia dentro de la escuela es un problema anterior al uso de redes informáticas para acosar, abusar y atemorizar a otros, y, aun cuando el ciberbullying posee características propias que tienen que ver con las posibilidades de comunicación que otorgan las TIC, sigue siendo una faceta particular de un fenómeno más amplio. Por esta razón es que suele entenderse el ciberbullying como una manifestación del bullying, con atributos particulares como, por ejemplo, la posibilidad de mantener el anonimato por parte del agresor, que lo sitúa en una posición de ventaja para llevar a cabo un acoso o maltrato persistente en el tiempo.

Ahora bien, el concepto de ciberbullying hereda una dificultad importante que proviene de tratar la violencia escolar como sinónimo de bullying: se tiende a reducir la violencia existente a una expresión en particular, relacionada con la intimidación y el maltrato a través del "matonaje". Por supuesto que esta reducción tiene consecuencias significativas.

En primer lugar, "la violencia puede ser significada como una forma de relación interpersonal, de convivencia, y no exclusivamente como una forma de maltrato" (Potocnjak \& Berger, 2011). Más aún, cuando esta forma de maltrato está restringida a la intimidación y al matonaje, se tiende a invisbilizar otras formas de violencia que pueden estar presentes en la escuela y que determinan el clima de convivencia y la manera como se establecen las relaciones interpersonales. Por ejemplo, la violencia que muchas veces la propia organización ejerce a través de normas, procedimientos y prácticas que fomentan la discriminación, el individualismo, la competitividad, el clasismo, la segregación, el autoritarismo o el abuso de poder, queda oculta tras los casos de bullying, que se convierten en el problema más notorio e impactante. Lo mismo ocurre con la violencia social a la que están expuestas muchas escuelas llamadas "vulnerables".

En este sentido, vale la pena considerar algunos de los trabajos llevados a cabo por Rosario Ortega, Eva Romera y Rosario del Rey (2009), que han investigado el problema del ciberbullying en Nicaragua. Estas investigadoras ofrecen un acercamiento a la situación de maltrato escolar existente en Managua, la Capital de un país considerado de bajos ingresos, con altos niveles de delincuencia. Mediante un cuestionario sobre convivencia, violencia y experiencias de riesgo que aplicaron a una muestra de 3.042 estudiantes, recogieron información en torno a la relación entre los roles que participan en los fenómenos de acoso y maltrato entre iguales (agresores, víctimas, agresores victimizados y espectadores) y las formas de manifestación de este tipo de violencia interpersonal. Tal vez una de sus conclusiones más significativas, es que las convenciones sociales creadas entre iguales, constituyen la materia prima de las situaciones de maltrato dentro de la escuela, ya que un importante porcentaje de niños y niñas que manifiestan conductas de violencia hacia otros, han sido también víctimas de situaciones de maltrato y de abuso de poder. Por supuesto que estas 
convenciones sociales no aparecen por sí solas, sino que son parte de un contexto social y cultural más amplio.

En segundo lugar, esta reducción induce a que otras formas de maltrato se vean bajo el prisma del acoso, del abuso de poder y del "matonaje". Especialmente, en el caso del ciberbullying está mirada, en muchas ocasiones, resulta forzada. Es común que estudiantes de bajo perfil, muchas veces tímidos, utilicen Internet para "vengarse" de compañeros o de compañeras que los agreden, los atacan o los humillan en la escuela. A veces, estas venganzas se realizan de formas bastante crueles, pero de manera ocasional, sin repetirse en el tiempo. ¿Diremos, en este caso, que se convierten en "matones" cuya fuerza consiste en la capacidad para ocultarse en el anonimato? ¿O es que acaso estas formas de maltrato no son violencia y, por lo tanto, no son importantes para que la institución educativa las tome en consideración? En cualquiera de los dos casos, comprender el maltrato escolar a partir del matonaje y la intimidación no ayuda a clarificar el problema.

En tercer lugar, y muy relacionado con el punto anterior, al tratar de comprender e intervenir situaciones de violencia escolar identificando víctimas agredidas, victimarios agresores y espectadores cómplices o indiferentes, inevitablemente, tenderemos a estigmatizar a determinados estudiantes y a contribuir a la fijación de conductas en ellos, asociadas a este tipo de roles. Además, estaremos influenciados por un sesgo que solo nos permite ver la expresión de la violencia circulando en una dirección: desde quien hemos identificado como victimario hacia quién hemos reconocido como víctima. Nos costará mucho, por ejemplo, visualizar situaciones en que la violencia va desde la víctima o desde el entorno, hacia el victimario. Por el contrario, tenemos el peligro de "naturalizar" las respuestas violentas hacia quienes hemos identificado como agresores, porque es lógico que a los victimarios hay que castigarlos o reprimirlos de alguna forma.

En cuarto y último lugar, el bullying o acoso escolar, suele abordarse a partir de la intimidación que ejerce un acosador hacia una víctima. La palabra Intimidación se refiere a causar o infundir miedo y el miedo es una emoción experimentada por alguien, por un sujeto. El miedo no existe en abstracto. Es una experiencia que alguien vive en función de algo que lo amenaza o lo atemoriza. Si no hay miedo, no puede haber intimidación. Por lo tanto, al entender el maltrato escolar a partir de la intimidación, implícitamente estamos abordando el problema de la violencia desde la perspectiva del sujeto atemorizado, es decir, desde la perspectiva de la "víctima" que experimenta miedo. Tal como lo describe María Isabel Toledo (2009):

La intimidación es el hostigamiento de un estudiante o grupo a otro estudiante o grupo de estudiantes. Se trata de una relación asimétrica de poder, en la cual el más débil es incapaz de responder la agresión. Pero también se define por su carácter repetitivo. Se trata de un particular tipo de interacción social, de un sistema de roles que se asumen en contextos específicos, aunque algunos de estos roles se tienden a fijar. Entonces, la intimidación corresponde a una acción situada donde participan intimidadores líderes, asistentes del intimidador, reforzadores del intimidador, defensores de las víctimas, testigos y víctimas. Ella está presente en todos los establecimientos educacionales y constituye un grave problema educacional.

Cabe, por tanto, la siguiente pregunta: la explicación de un fenómeno a partir de una emoción determinada, como el miedo que se deriva de la intimidación, ¿incide en la manera cómo dicho fenómeno es abordado, comprendido e intervenido? 
Pensamos que sí. De acuerdo a Humberto Maturana (2001), "las emociones son disposiciones corporales que determinan o especifican dominios de acciones". Cuando cambia la emoción, cambia el dominio de la acción.

\begin{abstract}
Al mismo tiempo, todos sabemos que cuando estamos en una cierta emoción hay cosas que podemos hacer y cosas que no podemos hacer, y que aceptamos como válidos ciertos argumentos que no aceptaríamos bajo otra emoción. Tomemos como ejemplo de lo dicho la situación siguiente: al llegar a la oficina uno declara que piensa pedir un aumento de sueldo al jefe, y la secretaria amiga dice: "no le pidas nada hoy porque está enojado, no te va a dar nada". ¿No es acaso lo que dice la secretaria una indicación de que ella sabe que la persona enojada solamente puede actuar de una cierta forma, no porque esté restringida de una manera absoluta, sino porque está en un dominio en el que sólo son posibles ciertas acciones y no otras? Así decimos también, que las cosas dichas con enojo tienen una potencia, un valor, o una respetabilidad distinta de aquellas dichas en la serenidad y en el equilibrio. ¿Por qué? No porque una cosa dicha en el enojo sea menos racional que una dicha en la serenidad, sino porque su racionalidad se funda en premisas básicas distintas, aceptadas a priori desde una perspectiva de preferencias que el enojo define. Todo sistema racional se constituye en el operar con premisas aceptadas a priori desde cierta emoción (Maturana, 2001).
\end{abstract}

Desde la emoción de miedo de una víctima, una situación de violencia será susceptible de entenderse a partir de la interacción entre víctimas débiles, que se enfrentan a victimarios fuertes, crueles, amorales e incapaces de ponerse en el lugar de los demás, y que están rodeadas de espectadores indiferentes a las peticiones de auxilio de las personas vulnerables. Esto no quiere decir que las situaciones descritas como bullying, sean inventadas o ficticias. Lo que significa, es que, simplemente, de manera implícita, estamos asumiendo un punto de vista que no detectamos, originado a partir de la emoción de temor, que determina la manera en que damos estructura y racionalizamos el fenómeno de la violencia.

\title{
El aprendizaje de la violencia.
}

Maritgen Potocnjak, Christian Berger y Tatiana Tomicic (2011), en torno a la violencia que se genera dentro de la escuela, afirman que:

Lo que configura a un acto como violencia entre pares, desde la perspectiva de los actores, es la experiencia de violencia, victimización y daño, pero no la conducta en sí. Por ejemplo, Troop-Gordon y Ladd (2005) muestran que hombres y mujeres pueden interpretar de maneras distintas su experiencia de victimización .

Para estos investigadores, lo fundamental de la violencia está en la experiencia de aquel que recibe algún daño. El problema, sin embargo, es que no todo acto que produce daño puede ser considerado violento, por parte de quién lo lleva a cabo. Hay muchas situaciones en que las personas, más allá de su voluntad y del cuidado que ponen en sus acciones, producen daño en otros, por ejemplo, cuando ocurre un 
accidente: una persona que conduce un vehículo, de pronto sufre un ataque, pierde el control de la máquina y choca con otro conductor. La persona que perdió el control del vehículo, lo más probable es que deba responder por su desafortunada acción, sin embargo, es difícil sostener que han actuado con violencia. El problema es más complejo de lo que parece. Las conductas, en sí mismas, no son suficientes para definir lo que es y no es violencia, porque, además, una misma conducta puede ser experimentada e interpretada de diferentes maneras por distintas personas.

Ahora bien, si la violencia no está en la conducta en sí misma, ni tampoco, exclusivamente, en el daño que puede generar, ¿qué es lo que la define? Veámoslo a través del siguiente ejemplo: un médico utiliza un bisturí para abrir la piel y cortar la carne de una persona. Puede extraer la vesícula de un enfermo que llega con intensos dolores al hospital. Por supuesto que no se trata de un acto de violencia. Todo lo contrario, su acción responde a la intención de ayudar, de sanar a alguien. Aun cuando una operación no tenga éxito y el resultado sea la muerte de una persona, si los médicos han procedido de manera responsable y han hecho todo lo que está en sus manos, en ningún caso podremos decir que se trata de violencia. Por el contrario, si un sujeto clava un cuchillo en el estómago de alguien, con la intención de que sufra y muera, será un criminal y la acción que realiza, un deplorable acto de violencia, aun cuando el afectado sobreviva. La violencia, por lo tanto en primer lugar, depende de la intencionalidad con que se hace algo y no solamente de los resultados o de las consecuencias que producen las acciones.

Podemos, sin embargo, observar las acciones y los resultados de las acciones de otras personas, pero su intencionalidad sólo podemos interpretarla. Lo mismo ocurre con nuestra propia intencionalidad: los demás pueden ver nuestros actos y sus consecuencias, pero la intencionalidad con que hacemos las cosas es parte de nuestra experiencia y los demás no pueden sentirla ni experimentarla. Sólo pueden suponerla e interpretarla.

Aquí encontramos el primer problema para aproximarnos al concepto de la violencia: aquello que la define (la intencionalidad) no es un hecho ni un comportamiento objetivo, sino que depende de la manera en que los sujetos damos sentido y significado a nuestras acciones, y de la manera como los demás interpretan ese significado. Esto no quiere decir que la violencia sea un fenómeno que habita en el relativismo absoluto de la subjetividad, sino, más bien, que se encuentra en el campo de lo intersubjetivo, como muchos otros fenómenos a partir de los cuales los seres humanos nos desenvolvemos e interactuamos socialmente, como, por ejemplo, las creencias, los valores e, incluso, el lenguaje. En este sentido, los signos en el lenguaje son creaciones colectivas que trascienden al sujeto, sin embargo, el sentido y el significado que se otorgan a dichos signos dependen de cada sujeto que utiliza ese lenguaje.

¿Cuál es, entonces, la intencionalidad que caracteriza a la violencia? Revisemos algunas definiciones y nociones de violencia.

Para Domenach,

La violencia es específicamente humana por cuanto es una libertad (real o supuesta) que quiere forzar a otra. Llamaré violencia al uso de una fuerza, abierta u oculta, con el fin de obtener de un individuo, o de un grupo, algo que no quiere consentir libremente. (1981, p.36)

Para Vicente Hueso,

La violencia pretende dañar humana y materialmente $\mathrm{y}$, a veces con asiduidad. Normalmente cuando ésta surge origina una espiral de violencia o si se quiere una dialéctica entre defensa y revancha. Esta 
espiral se convierte, en palabras del autor, en un metaconflicto, o por así decirlo, en una metástasis en términos médicos, extendiéndose más allá de los objetivos que hay que preservar y destruir originariamente. De esta forma, un conflicto puede adquirir una vida eterna, creciendo y menguando, desapareciendo y reapareciendo (2000)

Así mismo, para Johan Galtung (2003), uno de los referentes contemporáneos en el área de las ciencias sociales, violencia significa dañar y/o herir. La violencia es entendida como cualquier tipo de acción o circunstancia humana que genera daño sobre las personas y sobre su entorno. Si es en una situación de violencia es posible reconocer a un emisor o un agresor, hablaremos de violencia directa (física o psicológica). Si no es posible identificarlo, se trata de violencia indirecta o estructural. La construcción de la paz consiste, precisamente, en reducir la violencia y evitarla o prevenirla. La violencia simbólica o cultural, es una tercera forma de violencia que consiste en mentalidades, creencias, valores y modos de pensar que se orientan a hacer daño, convirtiéndose en sentido común que invita a la violencia directa y legitiman la violencia estructural. Ejemplos de la violencia simbólica son, el clasismo, el racismo, el machismo, la homofobia y la intolerancia religiosa, entre otros.

El común denominador de las distintas expresiones de la violencia es, en primer término, la negación del otro (o de los otros) como un ser humano legítimo. Esta negación deriva en disposiciones afectivas como el odio, el rechazo, el desprecio, la indolencia, el deseo de venganza, etc. y puede desencadenar acciones para agredirlo, dañarlo, destruirlo, discriminarlo, humillarlo, utilizarlo y cosificarlo o, simplemente, ignorarlo en sus necesidades y derechos. Esta es la intencionalidad que caracteriza a la violencia y que es posible reconocer tanto en la violencia directa, como en la indirecta y la cultural, en la nomenclatura de Galtung. En el caso de la violencia indirecta y de la violencia cultural, la negación del otro como un ser humano legítimo, está incorporada estructuralmente de manera social y colectiva.

En nuestra sociedad actual que, por una se basa en la tradición judeocristiana y su imperativo conceptual de la Ley, por otra, exacerba el individualismo y, por otra, ha hecho predominar el darwinismo social en su ordenamiento económico y político, la violencia es parte consustancial de las relaciones personales y colectivas (JiménezBautista, 2012). Una parte importante de los vínculos sociales e interpersonales que se establecen en la actualidad, están sustentados en la violencia y se han convertido en algo tan habitual, que es necesario hacer un esfuerzo especial para dilucidar el papel que está jugando esta violencia, en todas sus dimensiones.

Probablemente, es por esta razón que el determinismo biológico, como una concepción que plantea que la violencia es "natural" en el ser humano, ha tenido una influencia tan grande en nuestra manera de entender las cosas, incluso en el pensamiento que incorporan diversas disciplinas científicas. Sin embargo, como lo ha señalado en varias oportunidades la UNESCO, y en especial, a través del Manifiesto de Sevilla en mayo de 1986, la violencia no es "innata", sino que se "aprende" a lo largo de la vida. Si bien, hay ciertas características de la personalidad humana que se heredan genéticamente e inciden en el carácter, el comportamiento violento es una conducta que aparece condicionada por el entorno social (económico, político y cultural) donde se encuentran las personas.

La violencia posee un carácter multifacético y puede ser ubicada en diversas escalas (micro, meso, macro o mega) y en distintos ámbitos (individuos, familias, grupos, instituciones, civilizaciones). En el ámbito de la escuela, puede manifestarse a través de las relaciones entre sus actores: alumnos, docentes, padres, directivos y presentarse con características que le son propias. Es evidente que la escuela no 
enseña la violencia con una intencionalidad educativa, sin embargo, quiéralo o no, está relacionada con ella, ya sea, a través de lo que algunos expertos llaman el currículum oculto o, incluso, mediante condiciones que van más allá de ella misma y que tienen que ver con su funcionamiento dentro de una estructura social mayor. Por ejemplo, en el caso de Chile, la escuela reproduce la misma segregación de clases que existe en la sociedad chilena. Es así como, según cifras publicadas por la Organización para la Cooperación y el Desarrollo Económico (OCDE) en el año 2011, Chile ha sido identificado como el país con mayor segregación socio-económica a nivel escolar entre los países que integran la OCDE.

\section{La violencia escolar entendida desde la identidad.}

De acuerdo a un estudio llevado a cabo por Michael Boulton y Kerry Underwood (1992), en el cual se analizaron las atribuciones emocionales de niños y niñas directamente implicados en bullying, los agresores tendían a actuar en función de la reputación social y del refuerzo que obtenían de los espectadores, ya que su comportamiento les entregaba un papel dominante dentro del grupo. En este mismo sentido, para Berger y Rodkin (2011), la violencia en la escuela cumple una función adaptativa en la forma como los estudiantes se vinculan entre sí, y es utilizada como un mecanismo de integración de acuerdo a perfiles que se establecen para participar en el grupo.

Cabe, por lo tanto, preguntarse: ¿por qué razón el maltrato entre compañeros se traduce en una mejor reputación social para aquellos que demuestran capacidad para violentar a otros? En relaciones interpersonales marcadas por la violencia, ¿qué incidencia tiene la configuración y valoración de roles que se lleva a cabo dentro de un grupo? Si entendemos la violencia como negación del otro, ¿qué importancia tiene entender aquellas formas de ser y de expresarse que se aceptan y aquellas que se rechazan, dentro de un contexto de relaciones interpersonales? Por último, ¿El maltrato escolar es un problema que tiene que ver, exclusivamente, con la personalidad y los impulsos de alumnos agresivos y poco empáticos o se trata, más bien, de una manera de socializar dentro de la escuela? Todas estas preguntas nos conducen a la necesidad de dilucidar, en primer término, los conceptos de identidad y rol.

El concepto de "identidad" proviene de la palabra latina "identĭtas, -ātis." que, a su vez, proviene de "idem", un pronombre formado por id más la partícula -dem: "uno mismo, el mismo". Su significado posee una dualidad: por una alude a las características que nos hacen percibir que una persona es diferente a las demás; por otra parte, estas características se encuentran presentes en otras personas, de tal modo que gracias a ellas podemos compararlas. La identidad sólo describe atributos que se comparten con otras personas. Por ejemplo, todos tenemos un nombre, un apellido, un número de identificación, etc. Gracias a estas propiedades o atributos, que se asignan a cada individuo, cada individuo se diferencia del resto y es posible "identificarlo". Por su parte, entenderemos los roles como funciones o papeles específicos que alguien desempeña en un contexto determinado. Kluckhohn y Murray (1948) consideran que la identidad es la combinación específica de los roles de cada individuo.

La identidad, por lo tanto, al señalar características que compartimos con otras personas, siempre es en relación a los demás. A través de la identidad, se ocupa un lugar en el mundo, se desempeña un rol, se establecen los límites de la acción y se encauza dicha acción. La identidad, "identifica" a un individuo o a un conjunto de individuos y lo(s) coloca en relación con los demás.

Esto significa que las propiedades o los atributos vinculados a la identidad son propiedades o atributos "para" los demás, para una mirada externa que se termina 
incorporando como una mirada propia. Tenemos un nombre, un apellido, una cédula de identidad, es decir, un número que nos identifica en la sociedad; tenemos, además, ciertas características físicas, como el color de pelo, de ojos, la estatura, la contextura, los rasgos faciales, el tono de voz. Poseemos, también, una historia, un "currículum", que nos emplaza en una clase social, nos otorga una ciudadanía, nos permite disponer de ciertos recursos económicos y, entre otras muchas cosas, nos posibilita o nos impide desempeñar determinados roles. Todas estas características o propiedades que nos identifican, son vistas por los demás y gracias a ellas, los demás nos reconocen, nos asignan un lugar en la sociedad y en cada grupo en el que nos desenvolvemos.

Por otra parte, hemos dicho que la violencia es, en términos generales, la negación del otro, y que esta negación deriva en disposiciones emocionales y en acciones orientadas a producirle daño (o, también en acciones orientadas a desampararlo cuando sufre daño). Sin embargo, ¿quién es este "otro"? Este "otro" es lo que se identifica y reconoce de él, en un contexto determinado. Por supuesto que esta identificación no se da en abstracto: siempre se produce en función de alguien que, individualmente o de manera colectiva, lo observa, lo percibe y, en última instancia, se relaciona con él. Para el matón de un curso, este "otro" puede ser el estudiante tímido al que vale la pena humillar para obtener reconocimiento de los pares; para el transeúnte que en la mañana va a su trabajo, este "otro" puede ser un niño que duerme en la calle y al que es preferible esquivar para evitar molestias; para el automovilista apurado este "otro" puede ser la señora a la que lanza todo tipo de improperios porque está utilizando la pista en la que él conduce. Sin embargo, el estudiante tímido tendrá un conjunto de otros roles y características que configuran su identidad: podrá ser hijo, hermano, primo, vecino, pasajero frecuente en el autobús, internauta, miembro de un grupo de Facebook interesado en los extraterrestres. El matón del curso conoce al estudiante tímido y se relaciona con él, a partir de una de las dimensiones de su identidad: aquella mediante la cual socializan en la escuela. De la misma forma, el niño vagabundo será tratado de manera muy distinta por el transeúnte, si sus identidades los vinculan como parientes directos; o la señora del automóvil, que es agredida por utilizar una pista, será tratada de otra manera si es la vecina o la jefa del conductor que viaja apurado.

Tenemos, entonces, como primer punto, que la violencia, como negación del "otro", niega, en contexto, determinados aspectos relacionados con la identidad y los roles de este "otro". Lamentablemente este "otro" es uno solo, y si en la calle un racista dispara y asesina a un muchacho de rasgos indígenas, matara a la persona completa, con sus diferentes roles y características de identidad.

Otro elemento a tomar en consideración es que, al ser la identidad y los roles asociados a ella, construcciones intersubjetivas, la violencia, como negación de ciertos aspectos de la identidad del otro, es, también, intersubjetiva. ¿Qué quiere decir esto? Primero, que el racismo, el machismo o la homofobia, se basan en contenidos, valores y creencias que están presentes en la sociedad y en la cultura y que determinados individuos y grupos incorporan en su visión del mundo. En segundo lugar, si lo llevamos a un plano más específico, de relaciones interpersonales, algunos individuos podrán (o no) tender cierta predisposición a la agresividad, a la impulsividad o a la falta de empatía con los demás. Sin embargo, es una simplificación de las cosas sostener que la violencia es producto de estas pulsiones "naturales" en el ser humano. La violencia se produce en contextos de relación, donde las personas ocupan roles y configuran sus identidades. Esto no sólo se aplica a grupos de personas conocidas y familiarizadas. También se da en el ámbito de relaciones esporádicas, donde los sujetos no se conocen o apenas se conocen. Por ejemplo, después de un partido de fútbol que termina en una batalla campal entre barras bravas, los sujetos de un bando no conocen a los del otro. Basta con que se reconozcan como del bando opuesto, para 
descargar toda su agresividad sobre el individuo que está al frente. Lo mismo ocurre con la guerra: es suficiente saber que el otro es el enemigo, para querer destruirlo. En la escuela, donde los sujetos sí se conocen y configuran una historia en común, el o los matones de un curso actúan en el marco de roles e identidades que han sido construidas en el tiempo y que tienden a fijarse. Roles e identidades que se definen entre los estudiantes, pero también en el contexto de las normas (explícitas e implícitas), los valores, las costumbres, las prácticas institucionales y un tipo de relación con la comunidad adulta de la escuela.

\section{Ciberbullying y violencia escolar a través de las nuevas tecnologías}

El ejercicio de la violencia a través de medios virtuales, lejos de aminorar o reducir las connotaciones negativas que se explicitan en la práctica de la violencia directa situada en un espacio material real, permiten la extensión del ser físico más allá de los límites de la piel (Derrik de Kerckhove, 1999), disolviendo inclusive, los propios mecanismos de defensa psicológica y físicas, que serían posibles de poner en juego cuando se mantienen las delimitaciones tradicionales de una interacción humana inmediata. Cuando este plano de interacciones humanas basadas en el ejercicio de la violencia, se traslada y se sustenta en una relación virtualizada, resulta aún menos indemne la posición del sujeto que es objeto de la victimización. Las características de instantaneidad, sumado la capacidad de masificación y de exposición que provee el fenómeno del ciberbullying, intensifican el sentido y el significado de la agresión, esto, además, en la incapacidad de la victima de revertir o contener el fenómeno de agresión en la red, como sucede en la mayoría de estas situaciones. El atentado hacia los límites de la privacidad, así como la falta de control respecto de lo que sucede, simboliza en el ciberbullying, la mayor vulneración al sentido y al desarrollo de la identidad.

De este modo, tanto el desarrollo y el despliegue de dicha identidad, que se proyecta en función de cómo logramos interpretar el mundo, a partir de los significados que otorgamos a los sucesos vitales y a la lectura que hacemos de lo que observamos de nosotros mismos en "el otro", se ve fuertemente atomizado, cuando se pierde el espacio de la legitimación social, aún en ausencia de daño físico real. Hoy en día el uso de las TIC, posibilita que esta construcción dialéctica de la identidad del sujeto (Berger Luckman, 1988), se encuentre fuertemente condicionada bajo nuevos parámetros, en donde no es posible disociar la "realidad" de la "realidad virtual". Así la arquitectura y la fluidez de esta identidad, asociada a los roles que ejercemos dentro de nuestra red familiar, escolar y social, emergerán por un lado desde este escenario tecnologizado e interconectado, siendo, por ende, a la vez susceptible a los daños provocados a nuestro rol dentro de una determinada red virtual.

En este sentido, el traslado de la violencia escolar, a las plataformas virtuales, evidencia no solo, la utilización funcional de un nuevo medio para ejercer formas de agresión, de discriminación y de intolerancia entre los alumnos, las cuales han estado presentes a lo largo de la historia de la institución escolar, sino que viene a proyectar además, que en el mundo actual, la configuración de nuestro espacio relacional ha adquirido las connotaciones y las propiedades distintivas otorgadas por este espacio virtual, que siendo inclusive, más volátil y flexible en la forma en que posiciona nuestro rol como usuarios de una red, desborda sin duda, el ámbito de las posibilidades de control, ya que anteriormente, el ejercicio de la permanencia y estabilidad de la identidad del sujeto, estaba contenida en su cuerpo físico (Weber Mitchell, 2008). Ahora, la configuración de estas nuevas formas de identidades sostenida en la realidad virtual, queda supeditada a una lógica que se sitúa y deambula entre lo virtual y lo corpóreo-presencial. 
De este modo, en la actualidad la forma y los espacios respecto a cómo establecemos la comunicación afectiva, familiar, escolar, laboral, la manera en como conocemos y reencontramos a otras personas, el intercambio de diversos tipos de información y contenidos, y en definitiva cómo se gestiona el actuar personal, institucional y social está contenida en la utilización de las TIC, ya sea sincrónica o asincrónicamente. Así la virtualización de nuestra identidad y de nuestra acción en el mundo a través del uso de estas tecnologías, nos provee de espacios y medios que fácilmente pueden llegar a sucumbir en lo iatrogénico, ante la posibilidad cierta de poder ser utilizadas para negar y violentar la identidad de otros, y así mismo, ante la posibilidad cierta de poder ser también violentados, de manera mucho más sofisticada, destructiva y deshumanizada. Por otro lado, las formas de violencia que se pueden poner de manifiesto a través de las redes como en las situaciones de ciberbullying, en primer siempre, compromete y agravia de igual forma la identidad de los sujetos en sus círculos personales y sociales directos anclados en el mundo real. De hecho, en los contextos escolares, es habitual que esta forma de agresión emane y se circunscriba a los propios compañeros de clase, quienes utilizan los medios tecnológicos para intimidar, rechazar, insultar o excluir a un alumno/a o grupo de alumnos/as, a través de la utilización de internet, telefonía celular u otro medio tecnológico, ya sea en formato de texto, videos, chats, fotografías, mensajería instantánea, correos electrónicos, creación de páginas web, entre otros (Pérez, Astudillo, Varela \& Lecannelier, 2013). En esta línea, Garaigordobil (2014) describe las formas en que el ciberbullying se manifiesta:

1. Subir a Internet una imagen real o diseñada mediante fotomontajes, publicar datos delicados o cosas que pueden perjudicar o avergonzar a la víctima.

2. Subir vídeos en una red social, donde se muestre una agresión física a una persona (happy slapping).

3. Telefonear a una persona con la intención de causarle temor, miedo, inseguridad o para proferirle insultos y/o amenazas.

4. Incorporar a una persona con foto incluida en algún sitio de la web, en donde se vota en base a un calificativo negativo, por ejemplo, la persona más fea o menos inteligente.

5. Excluir a una persona, impidiéndole participar en una red social específica.

6. Crearle a una persona un perfil falso y utilizarlo para publicar información o confesiones personales, demandas explícitas de contactos sexuales, o cosas similares.

7. Publicar en foros o chats, comentarios ofensivos o agresivos usurpando la identidad de otra persona con la intención de orientar reacciones negativas contra ella.

8. Acceder de manera ilegítima a las cuentas online de la víctima, enviando mensajes negativos o crueles hacia otras personas, para producir menoscabo a los contactos de la víctima.

9. Provocar de manera malintencionada a una persona en sitios web, que utilizan a una persona como moderador (chats, juegos online, comunidades virtuales, etc.) para conseguir una reacción violenta de esta, para que una vez denunciada o evidenciada, suponga su exclusión.

10. Hacer circular rumores sobre una persona, que impliquen un comportamiento reprochable u ofensivo, con la intencionalidad de que otros tomen represalia o asuman una respuesta de acoso.

11. Usurpar y cambiar las claves de correos electrónicos u otros sitios, de forma que la persona propietaria no pueda posteriormente acceder a estos.

12. Inscribir la dirección de correo electrónico de una persona, en determinados 
sitios para que luego sea víctima de spam, de contactos con desconocidos.

13. Enviar amenazas por correo electrónico o mensajería celular, hostigar y acosar a la víctima en los sitios de Internet que utiliza de forma habitual, mediante el envió reiterado de insultos o amenazas.

Podemos comprender, a partir de esta descripción que la intencionalidad existente en el ejercicio de la agresión hacia otro, se encuentra directamente o indirectamente referida a la provocación de un daño a su identidad, ya sea a partir de inducirle miedo (intimidarlo), vergüenza, rechazo, menoscabo social o exponerlo a situaciones de malestar donde el sujeto pierde la posibilidad de conducción.

\section{Conclusión}

La violencia en nuestra sociedad es un fenómeno pluridimencional y multicausal. Su análisis nos lleva, necesariamente a alejarnos de una racionalidad simplista y de una lógica lineal, para situarnos más bien en un reconocimiento intencional, que implica que para su comprensión, y las posibilidades de generar y proveer formas de abordaje, es decisiva la deconstrucción de las escenas y de los escenarios desde donde toma forma y se reproduce. En este sentido la violencia, no reconoce límites, su transversalidad en términos religiosos, sexuales, económicos, etarios, etc., ha sido uno de sus principales mecanismos de naturalización a lo largo de la historia. Las formas de violencia escolar, por ende, han de comprenderse, desde esta misma óptica.

Los pilares de la cultura posmoderna, basados en la automatización de los procesos y la virtualización de la realidad, y por ende los vínculos humanos, a través de la incorporación, y actualmente, dependencia a las nuevas tecnologías, han posibilitado ampliar aún más los márgenes posibles y la intensidad de la expresión de la violencia, en la escuela el ciberbullying como fenómeno, justamente ha venido a conjugar y evidenciar un desborde de los limites históricamente concebidos en ella, tanto desde su estructura física como simbólica, generando así una problemática de obtuso abordaje y de agudas consecuencias. De este modo, nos enfrentamos actualmente no solo a nuevas necesidades y desafíos en el ámbito educativo, sino además, nos sitúa en una panorámica global volátil y de aristas vertiginosas.

Al entender la violencia a partir de las identidades que hay en juego, asumimos un punto de vista integral, la desnaturalizamos y nos vemos en la necesidad de reflexionar en torno a los valores, las creencias y los estereotipos que se sostienen en un ámbito de relaciones humanas, más allá de la búsqueda de víctimas y de victimarios.

En este sentido, vale la pena mencionar la experiencia que ha tenido Finlandia mediante la implementación del programa KiVa, para combatir el acoso escolar y el ciberbullying, implantado en el $90 \%$ de los colegios de educación básica y con resultados sumamente exitosos. A diferencia de otros modelos que se basan, exclusivamente, en tratar a las víctimas y los acosadores, KiVa intenta transformar las normas que se incorporan en un grupo, influyendo, principalmente, en la actitud que asumen los testigos y generando conciencia en la importancia de las acciones del grupo (KiVa, 2014).

Para finalizar, la aplicabilidad de este punto de vista que, en resumen, plantea que la violencia escolar es el resultado de relaciones interpersonales que se sustentan en una construcción de identidades, requiere develar cuáles son, en concreto, estas identidades, los roles a partir de los cuáles se constituyen y los elementos que las 
caracterizan. Se trata de un tipo de estudio y de reflexión en contexto, con los actores que socializan en la escuela.

\section{Referencias}

Area, M. (2011). TIC, Identidad Digital y Educación. Cuatro reflexiones. Reencuentro, $\mathrm{N}^{\circ}$ 62, 97-99

Berger, C. \& Rodkin, P.C. (2011), Group Influences on Individual Aggression and Prosociality: Early Adolescents Who Change Peer Affiliations. Social Development.

Bifo, F. (2003). La Fábrica de la Infelicidad. Madrid: Traficantes de Sueños.

Boulton, M. J., y Underwood, K. (1992). Bully/victim problems among middle school children. British Journal of Educational Psychology, 62, 73-87.

Cite as: Elipe, P., Ortega, R., Hunter, S.C., \& Del Rey, R. (2012). Inteligencia emocional percibida e implicación en diversos tipos de acoso escolar (Perceived emotional intelligence and involvement in several kinds of school bullying). Psicologia Conductual/ Behavioral Psychology, 20(1), 169-181.

Gobierno de Chile, Ministerio del Interior (2009). Tercera Encuesta Nacional de Violencia en el Ámbito Escolar. Santiago; División de Seguridad Pública. Disponible en

http://www.seguridadpublica.gov.cl/filesapp/presentacion_violencia_escolar_2009_w eb.pdf

Del Barrio, C., Martín, E., Almeida, A. \& Barrios, A. (2003). Del maltrato y otros conceptos relacionados con la agresión entre escolares, y su estudio psicológico. Infancia y Aprendizaje, 26, 9-24

Domenach, J. (1981). La Violencia y sus Causas. París: Editorial UNESCO.

Galtung, J. (2000). La transformación de los conflictos por medios pacíficos. En V. García, Cuadernos de estrategia (pp. 125-159). España: Ministerio de Defensa: Instituto Español de Estudios Estratégicos

Galtung, J. (2003). Paz por medios pacíficos. Bilbao: Gernika Gogoratuz

Garaigordobil, M. Prevalencia y consecuencias del cyberbullying: una revisión. Revista International Journal of Psychology and Psychological Therapy. 11 (2), 233254.

Gutiérrez, V. et al (2009). Influencia de la intimidación (bullying) y la relación profesor - estudiante sobre el rendimiento en PISA 2009: un estudio multinivel. Fondo de Investigación y Desarrollo En Educación - FONIDE. Departamento de Estudios y Desarrollo. División de Planificación y Presupuesto. MINEDUC

KiVa International (2014). KiVa Anti-Bullying Program. Descargado el 03 de enero de 2016, de http://www.kivaprogram.net/

Kluckhohn, C., \& Murray, H. A. (1948). Personality in nature, society, and culture. New York: Knopf.

Maite Garaigordobil Landazabal (2014). Cyberbullying: Una nueva forma de violencia entre iguales a través de los medios electrónicos. Revista Padres y Maestros. $\mathrm{N}^{\mathrm{o}} 357,34-40$. 
Maturana, H. (2001). Emociones y Lenguaje en Educación y Política. Santiago de Chile: Editorial Dolmen.

Menay-López, L, De la Fuente-Mella, H. (2014). Plataformas comunicacionales del ciberbullying. Una aplicación empírica en dos colegios de la quinta región, Chile. Revista Estudios Pedagógicos XL (2), 117-133,.

Mora-Merchán, J. (2008). Cyberbullying: un nuevo reto para la convivencia en nuestras escuelas. Revista Información Psicológica. $\mathrm{N}^{\circ} 94,60-70$

Morillas, J. (2004). Qué es la violencia. En B. Molina y F. Muñoz (Ed). Manual de paz y conflictos. (pp. 225-248). España: Universidad de Granada.

Olweus, D. (1993). Bullying at school: What we know and what we can do. Oxford, UK: Blackwell.

Organización Mundial de la Salud (2002). Informe Mundial Sobre la Violencia y la Salud. Organización Panamericana de la Salud para la Organización Mundial de la Salud. Washington D.C.

Ortega, R. (2010). Agresividad Injustificada, Bullying y Violencia Escolar. Madrid: Alianza Editorial.

Ortega, R., Romera, E. (2011). Factores Asociados a la Implicación en Bullying: Un Estudio en Nicaragua. Revista Psychosocial Intervention, 20 (2), 161-170.

Ortega, R., Romera, E., Del Rey, R. (2009). La violencia escolar en la educación primaria de Managua: comprender el contexto para abordar la realidad. Revista Publicaciones. $\mathrm{N}^{\circ} 39,2009,31-41$

Pérez, J.C., Astudillo, J., Varela, J. \& Lecannelier, F. (2013). Evaluación de la efectividad del Programa Vínculos para la prevención e intervención del Bullying en Santiago de Chile. Revista Semestral de la Asociación Brasileña de Psicología Escolar y Educacional, SP. Volumen 17, Número 1, Rio de Janeiro, 163-172.

Potocnjak, M. y Berger, C. (2011). Una Aproximación Relacional a la Violencia Escolar Entre Pares en Adolescentes Chilenos: Perspectiva Adolescente de los Factores Intervinientes. PSYKHE 20 (2), 39-52

Rigby, K. (1996). Bullying in schools: And what to do about it. Brisol, PA; Jessica Kingsley Publisher.

Sánchez, V., Ortega, R., Menesini, E. (2012). La competencia emocional de agresores y víctimas de bullying. Anales de Psicología, Universidad de Murcia, 28, (1), 71-82

Saravi, G (2009). Juventud y sentidos de pertenencia en América Latina: causas y riesgos de la fragmentación social. Revista CEPAL, 98

Toledo, M. (2009). Relación entre intimidación (bullying) y clima en la sala de clases y su influencia sobre el rendimiento de los estudiantes. MINEDUC

World Health Organization (2014). Global Status Report On Violence Prevention 2014. Programa de las Naciones Unidas para el Desarrollo (PNUD) 